\title{
Cyclooxygenase inhibitors and the exercise-induced stress response
}

\author{
N Claassen (PhD) ${ }^{1}$ \\ J Snyman (MB ChB, MPharmMed, MD) ${ }^{2}$ \\ A Koorts (MSc) ${ }^{1}$ \\ H Nolte (MA (HMS) Biokinetics) $)^{3}$ \\ B Wagenaar (MSc) ${ }^{1}$ \\ M Kruger (Dip Nursing) ${ }^{1}$ \\ P J Becker (PhD) ${ }^{4}$ \\ M Viljoen (PhD, PhD) ${ }^{1}$ \\ ${ }^{1}$ Department of Physiology, University of Pretoria \\ ${ }^{2}$ Department of Pharmacology, University of Pretoria \\ ${ }^{3}$ Sport Research Institute, University of Pretoria \\ ${ }^{4}$ Biostatistics Unit, Medical Research Council, Pretoria
}

\begin{abstract}
Objective. This study investigated the effects of single dosages of the non-steroidal anti-inflammatory drug (NSAID) naproxen, and of the coxib, rofecoxib, on the exercise-induced stress response.

Design. Eight subjects (age $20.9 \pm 1.1$ years, weight 70.4 $\pm 3.9 \mathrm{~kg}$, height $170.9 \pm 6.7 \mathrm{~cm}$, body surface area $1.82 \pm$ $0.09 \mathrm{~m}^{2}$, body mass index $24.1 \pm 1.3 \mathrm{~kg} \cdot \mathrm{m}^{-2}$ ) took part in a double-blind, drug-placebo, cross-over design study. The experimental procedures were performed on 3 occasions on each volunteer, i.e. once on placebo, once on naproxen (single dose of $1000 \mathrm{mg}$ ) and once on rofecoxib (single dose of $50 \mathrm{mg}$ ).

Results. Mean post-exercise cortisol values were significantly higher than pre-exercise values with the subjects on placebo $(p=0.0365)$ and rofecoxib $(p=0.0208)$, but not on naproxen ( $p=0.0732$ ). Post-exercise oral temperatures were significantly higher than pre-exercise temperature values on placebo $(p=0.0153)$ and rofecoxib $(p=0.0424)$, but not on naproxen ( $p=0.5444)$.

Conclusion. The results of this study suggest a role for cyclooxygenase-1 (COX-1) in the exercise-induced cortisol and temperature response to exercise.
\end{abstract}

\section{CORRESPONDENCE:}

\section{Viljoen}

Department of Physiology

University of Pretoria

P.O. Box 2034

Pretoria

0001

Tel: 012-319 2140

Fax: 012-3211679

E-mail: mviljoen@medic.up.ac.za

\section{Introduction}

The exercise-induced stress response is an adaptational homeostatic shift intended to facilitate the demands put on the body by physical exertion. Most stressors, including heat, pain, trauma and exercise, stimulate prostaglandin synthesis. The mechanisms underlying this stimulation usually involve the release of pro-inflammatory cytokines with subsequent induction of prostaglandin synthesis which influences the central neuroendocrine regulatory mechanisms. ${ }^{14}$ This stressor-induced prostaglandin synthesis, in turn, initiates and modulates many aspects of the stress response such as neuroendocrine, autonomic nervous system, metabolic and temperature changes. The pathways through which prostaglandins are involved in the cortisol stress response include a direct influence on the release of corticotropin-releasing hormone $(\mathrm{CRH})$ and/or antidiuretic hormone $(\mathrm{ADH})$ from the paraventricular nucleus (PVN), and indirect stimulation of the PVN via the central noradrenergic neurons. ${ }^{8,14}$ Prostaglandins are further involved in the exercise-induced autonomic nervous system response ${ }^{7}$ and in the increase in body temperature and other metabolic adaptations. ${ }^{14}$ Several other aspects of the exercise-related neuroendocrine response, which are under control of the hypothalamus and the hypothalamo-pituitary axis, are also influenced by prostaglandins and other eicosanoids. ${ }^{8}$ It therefore speaks for itself that any medication that interferes with eicosanoid metabolism could have the potential to influence the exercise-induced stress response.

Eicosanoids are produced throughout the body under the influence of the cyclooxygenase (COX) activities of prostaglandin endoperoxide $\mathrm{H}$ synthases (PGHSs). Two PGHS isozymes exist, PGHS-1 and PGHS-2, commonly referred to as cyclooxygenase-1 or COX-1 and cyclooxygenase-2 or COX-2. COX-1 is thought to be constitutively expressed in virtually all tissues and to be 
involved in normal physiological functions such as protecting the kidney and the gastric mucosa and maintaining vascular homeostasis. ${ }^{14,18}$ COX-2, although initially thought not to exist constitutively, is now, in humans, known to be found as such in several types of tissues. ${ }^{10}$ The expression of COX-2 can further be induced in all tissues tested thus far, and its expression is stimulated in a rapid, exaggerated fashion by mitogens, growth factors and pro-inflammatory cytokines. ${ }^{10,18}$ It is at present assumed that COX-2, which is situated in the cerebral blood vessels and thus easily accessed by bloodborne cytokines for the induction of prostaglandins, could be responsible for many of the physical stress-induced adaptations. $^{14}$

COX inhibitors have an influence on eicosanoid metabolism and many of the effects of non-steroidal anti-inflammatory drugs (NSAIDs) are exerted through interference with this effect. ${ }^{20}$ COX inhibitors block the biosynthesis of prostanoids by inhibiting the COX activities of PGHSs. Their most generally known pharmacological effects are their anti-inflammatory, analgesic, antipyretic and platelet-inhibiting actions, but they are also said to be of value in the prevention of colon cancer (COX-2), as well as in slowing down the progression of Alzheimer's disease, ${ }^{18}$ and there are indications that they may be of benefit during exercise by decreasing postexercise muscle soreness and decline in strength. ${ }^{1}$

Although these drugs are known for their anti-inflammatory and analgesic effects there are health risks, such as an increase in gastrointestinal permeability, associated with the indiscriminate use of anti-inflammatory drugs. ${ }^{12}$ A number of other negative side-effects have been reported for NSAIDs, including potential ulcerogenic and nephrotoxic effects and there are indications that they may inhibit protein synthesis in skeletal muscle after eccentric resistant exercise, ${ }^{21}$ augment the exercise-induced increase in gastrointestinal permeability $^{12}$ and decrease muscle blood flow during exercise. $^{5}$

The considered-safer COX-2 selective inhibitors such as rofecoxib and celecoxib have been associated with ulcer-related side-effects and increases in cardiovascular morbidity. $^{13}$

NSAIDs may theoretically also influence the adaptive exercise-induced stress response through suppression of the synthesis of those prostaglandins that stimulate the fever response, the sympathetic nervous system and the hypothalamo-pituitary-adrenocortical axis. ${ }^{7,8,18}$

The frequent use of COX inhibitors by athletes is a wellknown occurrence. In view of their role in the development of the exercise-induced stress response, COX inhibitors could theoretically also be expected to have an effect on the performance of athletes. Despite evidence that aspirin (acetylsalicylic acid) can modulate the secretion of many hormones, there is as yet not conclusive evidence that any of the COX inhibitors has a marked effect on the neuroendocrine and metabolic homeostatic shift of exercise, ${ }^{8,16}$ and more research is needed.
Three broad classes of COX inhibitors are generally known. ${ }^{9}$ The best known is aspirin, synthesised from salicylic acid, which inhibits the activity of COX-1 by irreversibly acetylating a serine residue at position 529. The second class is the NSAIDs such as indomethacin and naproxen that inhibit both forms of the enzyme, i.e. COX-1 and COX-2. The third class is the selective COX-2 inhibitors, i.e. the coxibs such as rofecoxib. This study investigated the effects of the non-selective COX inhibitor naproxen (Naprosyn) and the selective COX-2 inhibitor rofecoxib (Vioxx) on the treadmillinduced stress response and on the recovery period.

Naproxen reduces the synthesis of prostaglandins by inhibiting COX activities of both isoforms of PGHSs, i.e. COX-1 and COX-2. The plasma half-life of naproxen is 12 - 15 hours and peak plasma concentrations are seen in about 2 hours. ${ }^{7,20}$ Rofecoxib is 50 times more selective in inhibiting the COX-2 isoenzyme. The plasma half-life is $10-17$ hours and peak plasma concentrations are seen in $2-3$ hours. ${ }^{9}$ Although referred to as a COX-2 inhibitor, an alternative term to COX-2 inhibitor would be 'COX-1 sparing'.

The aim of this study was to investigate the effects of single dosages of the NSAID, naproxen (1000 mg), and the coxib, rofecoxib (50 mg), on the expression of the treadmillinduced stress response as reflected in free salivary cortisol levels, heart rate, oral temperature and other metabolic parameters.

\section{Methods}

\section{Subjects and experimental design}

The effects of the 2 NSAIDs on the exercise-induced stress response were investigated in healthy students in a doubleblind, drug-placebo, cross-over design study in which each subject served as his/her own control. All tests started at the same time of the day and experimental conditions were similar for all exposures.

The mean age of the students $(N=8)$ was $20.9 \pm 1.1$ years, weight $70.4 \pm 3.9 \mathrm{~kg}$, height $170.9 \pm 6.7 \mathrm{~cm}$, body surface area $1.82 \pm 0.09 \mathrm{~m}^{2}$ and body mass index $24.1 \pm 1.3$ $\mathrm{kg} \cdot \mathrm{m}^{-2}$. During the first week of the study students had general medical examinations and those with either hypertension (> $140 / 90 \mathrm{mmHg}$ ), hypotension $(<100 / 60 \mathrm{mmHg}$ ), or any other pathological condition or risk factor, as well as students on chronic medication or performance-enhancing drugs were excluded. Students who developed any health complication such as colds or flu during the study were excluded. Other than the treadmill procedures of the study no strenuous physical activity was allowed over the 4 weeks each subject was involved in the study and subjects had to refrain from alcohol intake on the days before the experiments. During the same week the maximal oxygen consumption $\left(\mathrm{VO}_{2 \max }\right)$ of each student was determined, the procedures of the study explained and informed consent forms signed. Ethical clearance (Research Ethics Committee, Faculty of Health Sciences, University of Pretoria, clearance number: 
173/2002) was obtained for the study and all participants gave written informed consent.

\section{Testing and procedures}

The experimental procedure was performed on 3 separate occasions by each participant, i.e. once on placebo, once on naproxen (single dose of $1000 \mathrm{mg}$ ) and once on rofecoxib (single dose of $50 \mathrm{mg}$ ) swallowed 2 hours before initiation of the treadmill procedure. The drug or placebo was administered randomly by a third party in a cross-over design study performed double blind. A washout period of 7 days passed between the experimental days. Testing started at the same time of day in consideration of circadian rhythmicity.

The $\mathrm{VO}_{2 \max }$ tests comprised incremental increases in running speed at a constant gradient of $2^{\circ}$. The following protocol was used: 3 minutes at $8 \mathrm{~km} \cdot \mathrm{h}^{-1}$ and 2 minutes at $10,12,14,16$ and $17 \mathrm{~km} \cdot \mathrm{h}^{-1}$ respectively on a motorised treadmill with the subjects clothed in jogging shoes and light clothing. Expiratory oxygen, carbon dioxide and heart rates were continuously measured every 10 seconds during the tests (Schiller Cardiovit, CS100, Baar, Switzerland).

The study proper started the second week. On arrival for the experiment $(07 \mathrm{~h} 00)$ the heart rate monitor was fitted, oral temperature was taken sublingually for 3 minutes using a clinical thermometer and thereafter a salivary sample was collected for cortisol determination. This is referred to as time PRE1. Thereafter the drug or placebo was administered and a standardised breakfast consisting of toast and fruit juice was consumed. Two hours after administration of the drug/placebo the oral temperature was again taken and saliva collected for cortisol analysis (PRE2). The treadmill procedure consisting of 3 exercise intensities equivalent to $50 \%, 60 \%$ and $75 \%$ of maximal oxygen expenditure for 10,15 , and 5 minutes, respectively, was started immediately. Heart rate recordings were obtained continuously throughout the procedure. Salivary samples were again collected at the end of the exercise protocol (POST1), and at 30 minutes (POST2), 60 minutes (POST3) and 120 minutes (POST4) post-exercise. The collected salivary samples were centrifuged at $1000 \mathrm{~g}$ for 10 minutes at $4^{\circ} \mathrm{C}$ and aliquots of the supernatants were transferred to Eppendorf tubes and stored at $-20^{\circ} \mathrm{C}$ until analysis by enzyme-linked immunosorbent assay (ELISA) (DRG GmbH, Marburg, Germany). Oral temperatures were recorded at the same times salivary samples were taken.

\section{Statistics}

Data were analysed using the appropriate analysis of variance (ANOVA) for a three-period cross-over experimental design, with factors for treatment period, treatment, carry-over and subjects. The analyses were also done by adjusting for baseline values, i.e. baseline values were used as covariates. The covariates were not significant for any of the variables. Within treatments, the exercise-induced effect was assessed using the paired $t$-test after establishing that there was no carry-over effect between treatments. As the sample was relatively small the conclusions for the latter were confirmed using Wilcoxin's matched pairs signed-rank test. Differences were considered statistically significant when $p<0.05$. $^{19}$

\section{Results}

Table I (A-C) shows the means, standard deviations and $p$ values (one-way ANOVA) for cortisol, heart rate and oral temperature directly before the drug/placebo was administered (PRE1), 2 hours after drug/placebo administration, i.e. directly before initiation of the treadmill procedure (PRE2), immediately after completion of the treadmill procedure (POST1), 30 minutes after the treadmill procedure (POST2), 60 minutes after the treadmill procedure (POST3), and 2 hours after the treadmill procedure (POST4). No significant differences were seen between the values obtained on placebo and with either of the 2 NSAIDs, or between the values on naproxen and on rofecoxib. Table I (D) shows the peak values obtained during exercise for the metabolic parameters. No significant differences were found between the values obtained on the drugs and placebo or between those on the 2 drugs.

The exercise-induced response and the post-exercise recovery for the various parameters are recorded in Table I. It can be seen that the treadmill procedure served its function as physical stressor as typical stress responses were observed over the exercise period in all 3 groups. The mean post-exercise cortisol values (POST1) were significantly higher than the pre-exercise (PRE2) cortisol values with the subjects on placebo $(p=0.0365)$ and rofecoxib $(p=0.0208)$, but not on naproxen $(p=0.0732)$. There were, however, no significant differences between the exercise-induced increases in cortisol secretion (POST1 minus PRE2) with subjects on placebo, naproxen and rofecoxib ( $p=0.9055)$. The mean post-exercise heart rate (POST1) was significantly higher than the pre-exercise (PRE2) heart rate values with subjects on placebo $(p=0.0001)$, naproxen $(p=0.0001)$ and rofecoxib $(p=0.0001)$. No significant differences were found between the exercise-induced increases in heart rate in the 3 groups $(p=0.3841)$. The post-exercise temperature (POST1) was significantly higher than the pre-exercise (PRE2) temperature values on placebo $(p=0.0153)$ and rofecoxib $(p=0.0424)$, but not on naproxen $(p=0.5444)$. Once again no statistically significant differences were found between the exercise-induced responses on the 3 substances ( $p=$ 0.6701).

The mean post-exercise METS $\left(1 \mathrm{MET}=3.5 \mathrm{ml} \mathrm{O} \cdot \mathrm{kg}^{-1}\right.$. $\mathrm{min}^{-1}$; POST1) was significantly higher than the pre-exercise (PRE2) METS values on placebo $(p=0.0001)$, naproxen $(p=0.0001)$ and rofecoxib $(p=0.0001)$. The means of the oxygen expenditure at $75 \% \mathrm{VO}_{2 \max }\left(\mathrm{VO}_{2 / \mathrm{min}}\right.$ and $\left.\mathrm{VO}_{2 / \mathrm{kg} / \mathrm{min}}\right)$ were significantly higher than the pre-exercise expenditure on placebo $(p=0.0001$ and $p=0.0001)$, naproxen $(p=$ 0.0001 and $p=0.0001)$ and rofecoxib $(p=0.0001$ and $p=$ $0.0001)$. No statistical differences were, however, found in the increases $(\Delta)$ over the exercise period for METS, $\mathrm{VO}_{2 \mathrm{ml} /}$ $\min$ or $\mathrm{VO}_{2 \mathrm{~m} / \mathrm{min} / \mathrm{kg}}$ between subjects on placebo, naproxen or 


\begin{tabular}{|c|c|c|c|c|}
\hline & Variable & Placebo ( \pm SD) & Naproxen ( \pm SD) & Rofecoxib ( \pm SD) \\
\hline & PRE 1 & $7.6 \pm 3.7$ & $7.8 \pm 6.4$ & $7.4 \pm 3.6$ \\
\hline A. & PRE 2 & $1.8 \pm 1.5$ & $1.4 \pm 1.3$ & $1.1 \pm 0.6$ \\
\hline \multirow[t]{5}{*}{ Mean cortisol concentration (ng/ml) } & POST 1 & $3.8 \pm 2.8$ & $3.0 \pm 1.7$ & $3.8 \pm 2.4$ \\
\hline & POST 2 & $2.7 \pm 1.7$ & $3.2 \pm 1.3$ & $3.6 \pm 2.4$ \\
\hline & POST 3 & $1.4 \pm 1.0$ & $2.0 \pm 0.7$ & $2.1 \pm 1.2$ \\
\hline & POST 4 & $0.8 \pm 0.5$ & $1.7 \pm 1.2$ & $1.6 \pm 1.3$ \\
\hline & PRE & $87.8 \pm 8.6$ & $83.6 \pm 5.6$ & $83.7 \pm 10.1$ \\
\hline B. & $50 \%$ & $113.8 \pm 8.9$ & $112.6 \pm 12.1$ & $111.1 \pm 7.5$ \\
\hline \multirow[t]{4}{*}{ Mean heart rate (beats/min) } & $60 \%$ & $148.1 \pm 21.2$ & $148.2 \pm 19.6$ & $156.2 \pm 15.5$ \\
\hline & $75 \%$ & $162.8 \pm 13.3$ & $169.8 \pm 13.1$ & $159.8 \pm 24.2$ \\
\hline & POST & $120.2 \pm 18.0$ & $122.9 \pm 25.2$ & $114.5 \pm 11.8$ \\
\hline & PRE 1 & $36.5 \pm 0.2$ & $36.4 \pm 0.3$ & $36.2 \pm 0.3$ \\
\hline C. & PRE 2 & $36.5 \pm 0.2$ & $36.5 \pm 0.4$ & $36.2 \pm 0.3$ \\
\hline \multirow[t]{5}{*}{ Mean oral temperature $\left({ }^{\circ} \mathrm{C}\right)$} & POST 1 & $36.7 \pm 0.3$ & $36.6 \pm 0.3$ & $36.6 \pm 0.3$ \\
\hline & POST 2 & $36.9 \pm 0.1$ & $36.6 \pm 0.4$ & $36.8 \pm 0.2$ \\
\hline & POST 3 & $36.7 \pm 0.3$ & $36.6 \pm 0.2$ & $36.7 \pm 0.2$ \\
\hline & POST 4 & $36.6 \pm 0.3$ & $36.6 \pm 0.2$ & $36.5 \pm 0.3$ \\
\hline & Rest & $1.1 \pm 0.6$ & $1.1 \pm 0.5$ & $0.9 \pm 0.8$ \\
\hline D. & $50 \%$ & $4.6 \pm 0.9$ & $5.1 \pm 0.9$ & $5.1 \pm 1.0$ \\
\hline \multirow[t]{4}{*}{ METS } & $60 \%$ & $6.9 \pm 1.2$ & $7.4 \pm 1.5$ & $7.6 \pm 1.4$ \\
\hline & $75 \%$ & $7.8 \pm 0.8$ & $8.6 \pm 0.9$ & $8.0 \pm 1.6$ \\
\hline & $\Delta \mathrm{MET}$ & $6.6 \pm 0.7$ & $7.5 \pm 1.0$ & $7.1 \pm 1.3$ \\
\hline & $\Delta \mathrm{VO}_{2 / \mathrm{min}}$ & $1.6 \pm 0.2$ & $1.9 \pm 0.3$ & $1.7 \pm 0.3$ \\
\hline \multirow[t]{4}{*}{ Exercise-induced response } & $\Delta \mathrm{VO}_{2 / \mathrm{kg} / \mathrm{min}}$ & $23.2 \pm 2.5$ & $26.4 \pm 3.4$ & $24.8 \pm 4.4$ \\
\hline & $\Delta$ Cortisol & $1.9 \pm 2.1$ & $1.6 \pm 2.1$ & $2.7 \pm 2.5$ \\
\hline & $\Delta$ Heart rate & $75.0 \pm 15.0$ & $86.2 \pm 14.9$ & $72.2 \pm 28.4$ \\
\hline & $\Delta$ Oral temp & $0.2 \pm 0.2$ & $0.1 \pm 0.4$ & $0.4 \pm 0.4$ \\
\hline \multicolumn{5}{|c|}{$\mathrm{SD}=$ standard deviation; $\mathrm{METS}=3.5 \mathrm{ml} \mathrm{O} \cdot \mathrm{kg}^{-1} \cdot \mathrm{min}^{-1}$} \\
\hline
\end{tabular}

rofecoxib ( $p=0.5122 ; p=0.4764 ; p=0.4433$ ).

No statistically valid differences were found over the recovery period between the results obtained on the 2 drugs or between the drugs and the placebo.

\section{Discussion}

In this study good stress responses were observed over the treadmill procedure for all parameters tested, indicating that the exercise regimen was correct. There were no significant differences between the absolute values over time for any of the stress parameters irrespective of whether the subjects were on placebo, naproxen or rofecoxib. Significant increases were found for all stress parameters over the exercise period (POST1 v. PRE2) when subjects were on placebo and on rofecoxib, respectively. However, the pre-post increases in cortisol concentrations and oral temperatures were not statistically significant with subjects on naproxen. Naproxen, as previously mentioned, belongs to the second class of antiinflammatory drugs that inhibit both forms of the enzyme, i.e. COX-1 and COX-2. Rofecoxib, on the other hand, belongs to the third class, i.e. the selective COX-2 inhibitors. The fact that the non-selective COX inhibitor, naproxen, but not the selective COX-2 inhibitor, rofecoxib, had an inhibiting effect on the cortisol and temperature response is an indication that the suppressive effects may be mediated by suppressing COX-1 activity.

The results of this study are unexpected as one would have thought that rofecoxib rather than naproxen would have had the suppressive effect. In theory, the slightly longer time to peak plasma concentration (Cmax) for rofecoxib than for naproxen might have been a confounding factor, which would have meant that the exercise could have been performed before peak plasma values were reached. This should, however, have been reflected in the recovery period. This 
was not the case as there were no statistical differences for any parameter between placebo, naproxen and rofecoxib over the post-exercise recovery period.

It is of interest that the lower cortisol response seen in this study with subjects on naproxen, compared with subjects on placebo, was also reported previously with aspirin. ${ }^{8}$ Further support for our findings, i.e. that COX-1 is involved in the exercise-induced stress response, is derived from indications that the COX-1 gene might be important in temperature regulation and the pyresis that occurs in the absence of infections, as opposed to COX-2 gene involvement in infection- and lipopolysaccharide-induced pyresis. ${ }^{4}$

Subsequent to completion of this study rofecoxib was voluntarily withdrawn worldwide on 30 September 2004 by Merck and Co. This followed the confirmation of previous results that the selective COX-2 inhibitor rofecoxib increases the relative risk for cardiovascular events such as heart attack and stroke. 3,6

Stress induces upregulation of COX-2 in endothelial cells and since COX-2 selective inhibitors such as rofecoxib have no antiplatelet activities (COX-1) the imbalance between prothrombotic eicosanoids ( $\mathrm{TXA}_{2}$ ) and vasodilatory and antiaggregatory $\mathrm{PGI}_{2}$ forms the theoretical basis for the reported adverse clinical outcome. ${ }^{2,17}$

Other COX-2 inhibitors such as celecoxib are still marketed for acute and chronic inflammatory pain. The association between celecoxib and adverse cardiovascular outcomes seems to be less pronounced. ${ }^{11}$ The reason for this is not clear but may be due to different pharmacokinetics compared with rofecoxib. Valdecoxib has also been associated with an increase in reinfarction rates in patients receiving the drug after a myocardial infarction. ${ }^{15}$ The potential for adverse cardiovascular events therefore seems to be related to the mechanism of action of COX-2 inhibitors.

The frequent use of NSAIDs by athletes before, during and after competitions raises the question whether it may have an influence on the exercise-induced stress response. This, and other investigations indicate that anti-inflammatory drugs may have an effect on the exercise-induced stress response and that the performance and perhaps even health of the athletes may be negatively affected by the use of these drugs. The results of this study therefore indicate that more care needs to be taken with the selection of NSAIDs if athletes have to use them during training and competitions. Coaches and physical conditioning specialists also need to be informed about the different physiological mechanisms that are influenced by NSAID drugs. This will enable them to give athletes proper advice with regard to the use of NSAIDs. It may further also help coaches and physical conditioning specialists to understand the development of certain unexpected exercise-induced stress responses if the athlete uses a specific NSAID.

\section{Conclusion}

Although the results of this study point towards a role for COX-1 in the exercise-induced cortisol and temperature re- sponse, more studies of this nature, as well as studies involving NSAIDs and endurance exercise, are urgently required to come to a better understanding of the exact role of COXs in the exercise-induced response and therefore of the potential effects of NSAIDs.

The authors would like to thank the subjects who participated in this study. The opinions, interpretations, conclusions and recommendations are those of the authors and do not constitute endorsement of the products used in this investigation. The data collection and technical efforts of $\mathrm{Dr}$ $\mathrm{M}$ Blom and Dr M van Wyk are also gratefully acknowledged.

\section{REFERENCES}

1. Baldwin AC, Stevenson SW, Dudley GA. Nonsteroidal anti-inflammatory therapy after eccentric exercise in healthy older individuals. J Gerontol A Biol Sci Med Sci 2001; 56: M510-3.

2. Belton O, Byrne D, Kearney D, Leahy A, Fitzgerald DJ. Cyclooxygenase-1 and -2-dependent prostacyclin formation in patients with atherosclerosis. Circulation 2000; 102: 840-5.

3. Bombardier C, Laine L, Reicin A, et al. Comparison of upper gastrointestinal toxicity of rofecoxib and naproxen in patients with rheumatoid arthritis. N Engl J Med 2000, 343: 1520-8.

4. Botting R. COX-1 and COX-3 inhibitors. Thromb Res 2003; 110: 269-72.

5. Boushel R, Langberg H, Gemmer C. Combined inhibition of nitric oxide and prostaglandins reduces human skeletal muscle blood flow during exercise. J Physiol (Lond) 2002; 543: 691-8.

6. Bresalier RS, Sandler RS, Quan H, et al. Cardiovascular events associated with rofecoxib in a colorectal adenoma chemoprevention trial. N Engl J Med 2005; 352: 1092-102.

7. De Meersman RE, Zion AS, Lieberman JS, Downey JA. Acetylsalicylic acid and autonomic modulation. Clin Auton Res 2000; 10: 197-201.

8. Di Luigi L, Guidetti L, Romanelli F, Baldari C, Conte D. Acetylsalicylic acid inhibits the pituitary response to exercise-related stress in humans. Med Sci Sports Exerc 2001; 33: 2029-35.

9. Fitzgerald GA, Patrono $\mathrm{C}$. The coxibs, selective inhibitors of cyclooxygenase-2. N Engl J Med 2001; 345: 433-42.

10. Gilroy DW, Colville-Nash PR. New insights into the role of COX 2 in inflammation. J Mol Med 2000; 78: 121-9.

11. Graham DJ, Campen D, Hui R, et al. Risk of acute myocardial infarction and sudden cardiac death in patients treated with cyclo-oxygenase 2 selective and non-selective non-steroidal anti-inflammatory drugs: nested case control study. Lancet 2005; 365: 475-81

12. Lambert GP, Broussard LJ, Mason BL, Mauermann WJ, Gisolfi CV. Gastrointestinal permeability during exercise: effects of aspirin and energycontaining beverages. J Appl Physiol 2001; 90: 2075-80.

13. Malhotra S, Shafiq N, Pandhi P. COX-2 inhibitors: a CLASS act or just VIGORously promoted. Medscape General Medicine 2004; 6: 1-9.

14. Moshonov S, Zor U, Naor Z. Prostaglandins. In: Fink G, ed. Encyclopaedia of Stress. vol. 2. San Diego: Academic Press, 2000: 266-372.

15. Nussmeier NA, Whelton AA, Brown MT, et al. Complications of the COX-2 inhibitors parecoxib and valdecoxib after cardiac surgery. $N$ Engl $\mathrm{J}$ Med 2005; 352: 1081-91.

16. Przybylowski J, Obodynski K, Lewicki $\mathrm{C}$, et al. The influence of aspirin on exercise-induced changes in adrenocorticotrophic hormone (ACTH), cortisol and aldosterone (ALD) concentrations. Eur J Appl Physiol 2003; 89: 177-83.

17. Ray WA, Stein MC, Daugherty JR, et al. COX-2 selective non-steroidal anti-inflammatory drugs and risk of serious coronary disease. Lancet 2002; 360: 1071-3.

18. Smith WL, De Witt DL. Cyclooxygenase inhibitors. In: Austen FK, Burakoff SJ, Rosen FS, Strom TB, eds. Therapeutic Immunology. Massachusettes: Blackwell Science, 2001: 117-31.

19. STATA for Windows (8.0). Stata Corporation, Texas, USA.

20. Dollery C, ed. Naproxen (sodium). In: Therapeutic Drugs. 2nd ed. vol. 2. Edinburgh: Churchill Livingstone, 1999: N31-N36.

21. Trappe TA, White F, Lambert CP, Cesar D, Hellerstein M, Evans WJ. Effect of ibuprofen and acetaminophen on postexercise muscle protein synthesis. Am J Physiol Endocrinol Metab 2002; 282: E551-6. 